NASA Technical Memorandum 103930

\title{
Vision-Based Range Estimation Using Helicopter Flight Data
}

Philip N. Smith, Banavar Sridhar, and Bassam Hussien

June 1992

(NASA-TM-103930)

RANGE ESTIMATION USING HELICOPTER

FLIGHT DATA (NASA) $20 \mathrm{P}$
N92-32424

Unclas

0117757 
NASA Technical Memorandum 103930

\section{Vision-Based Range Estimation Using Helicopter Flight Data}

Philip N. Smith, Banavar Sridhar, and Bassam Hussien

Ames Research Center, Moffett Field, California

June 1992

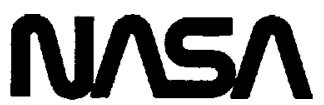

National Aeronautics and

Space Administration

Ames Research Center

Moffett Field, California 94035-1000 

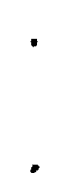

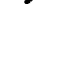




\title{
Vision-Based Range Estimation Using Helicopter Flight Data
}

\author{
Phillip N. Smith, Banavar Sridhar, and Bassam Hussien \\ NASA Ames Research Center \\ Moffett Field, CA 94035
}

\section{Abstract}

Pilot aiding during low-altitude flight depends on the ability to detect and locate obstacles near the helicopter's intended flightpath. Computer-visionbased methods provide one general approach for obstacle detection and range estimation. Several algorithms have been developed for this purpose, but have not been tested with actual flight data. This paper presents results obtained using helicopter flight data with a feature-based range estimation algorithm. A method for recursively estimating range using a Kalman filter with a monocular sequence of images and knowledge of the camera's motion is described. The helicopter flight experiment and four resulting datasets are discussed. Finally the performance of the range estimation algorithm is explored in detail based on comparison of the range estimates with true range measurements collected during the flight experiment.

\section{Introduction}

NASA Ames in conjunction with the U.S. Army has been investigating the use of passive range estimation with an imaging sensor to detect and locate obstacles which may represent a safety hazard to helicopters during low-altitude flight [1]. The obstacle information may be displayed directly to the pilot or used by an automatic obstacle-avoidance guidance system to enhance safety for near-terrain flight.

Several approaches for implementing passive range estimation have been investigated at NASA Ames $[2,3,4,5]$. In previous works, simulated and laboratory data have been used to demonstrate the performance of these algorithms. More recently, a helicopter flight experiment has been conducted to obtain samples of data that will be available from onboard systems during flight. The results of one passive range estimation algorithm using the helicopter data is presented in this paper to demonstrate the performance that can be expected in flight.

The range estimation algorithm presented here is based on a monocular sequence of images and on knowledge of the camera's motion. Optic flow measurements are derived by a feature-based method which tracks distinctive regions between images. An extended Kalman filter uses the measurements of optic flow and camera motion to recursively estimate range. The recursive nature of the Kalman filter provides an efficient mechanism for combining redundant measurements to successively refine the range estimates.

The helicopter flight experiment provides a realistic flight database including video images, motion state information, and camera calibration parameters. True range measurements were also obtained for evaluating the accuracy of range estimates. Straight-line and curved flight paths were flown over man-made and natural terrain.

The purpose of this paper is to present range estimation results using actual flight data, and to evaluate algorithm performance by comparison of range estimates with true range measurements. The range estimation algorithm is described in Section 2. focusing on the extended Kalman filter. Section 3 describes the helicopter flight experiment and the contents of the resulting flight database. Range estimation results obtained from four flight scenarios are the subject of Section 4. Conclusions and ideas for future work complete the paper.

\section{Estimation Algorithm}

Consider a helicopter-mounted camera that observes a point $P$ on a stationary object in the environment as shown in Figure 1. The image point $\{u, v\}$ corresponding to the point $P$ is given by the perspective 


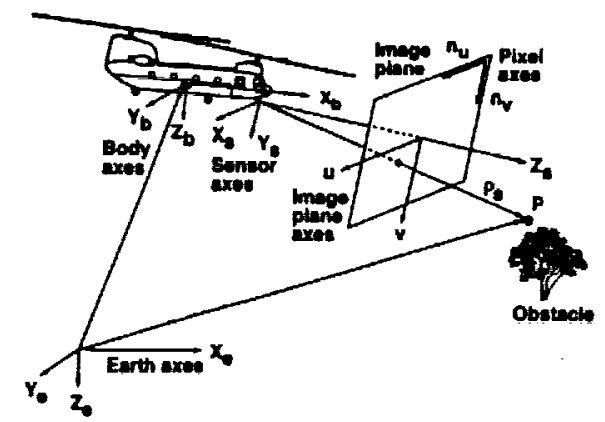

Figure 1: Geometry for Passive Range Estimation

projection equations as follows

$$
u=f x_{s} / z_{s}, v=f y_{s} / z_{s}
$$

where $x_{s}, y_{s}, z_{s}$ are components of $\rho_{s}$, the object's position relative to the camera, and $f$ is the focal length of the camera lens. As the camera moves, the image of $P$ will also move. If $P$ is assumed fixed in the Earth frame, the rate of change of $\rho_{s}$ in the camera's axes system can be determined using the Coriolis equation as follows

$$
\dot{\rho}_{s}=-V_{s}-\omega_{\mathrm{s}} \times \rho_{\mathrm{s}}
$$

where $V_{s}=\left\{V_{x s}, V_{y s}, V_{z s}\right\}$ and $\omega_{s}=\left\{\omega_{x s}, \omega_{y s}, \omega_{z s}\right\}$ are the camera's translational and rotational velocities with respect to the Earth frame. Differentiating the perspective projection equations with respect to time and substituting for $\dot{\rho}_{s}$ according to the above equation yields the well-known optic flow equations which relate camera motion, object motion in the image, and the object's range. The optic flow equations are as follow

$$
\begin{aligned}
\dot{u}= & \left(-f V_{x s}+u V_{z s}\right) / z_{s} \\
& +\frac{u v}{f} \omega_{x s}-f\left(1+\frac{u^{2}}{f^{2}}\right) \omega_{y s}+v \omega_{z s} \\
\dot{v}= & \left(-f V_{y s}+v V_{z s}\right) / z_{s} \\
& +f\left(1+\frac{v^{2}}{f^{2}}\right) \omega_{x s}-\frac{u v}{f} \omega_{y s}-u \omega_{x s} .
\end{aligned}
$$

Decomposing the optic flow into components due to camera translation $\left(\dot{u}_{t}, \dot{v}_{t}\right)$ and rotation $\left(\dot{u}_{r}, \dot{v}_{r}\right)$ gives

$$
\dot{u}=\dot{u}_{t}+\dot{u}_{r}, \dot{v}=\dot{v}_{t}+\dot{v}_{r}
$$

where

$$
\begin{aligned}
& \dot{u}_{t}=\left(-f V_{x s}+u V_{z s}\right) / z_{s} \\
& \dot{v}_{t}=\left(-f V_{y s}+v V_{z s}\right) / z_{s} \\
& \dot{u}_{r}=\frac{u v}{f} \omega_{x s}-f\left(1+\frac{u^{2}}{f^{2}}\right) \omega_{y s}+v \omega_{z s} \\
& \dot{v}_{r}=f\left(1+\frac{v^{2}}{f^{2}}\right) \omega_{x s}-\frac{u v}{f} \omega_{y s}-u \omega_{z s}
\end{aligned}
$$

With the optic flow equations, range to an object $z_{s}$ can be determined given measurements of the camera's motion state $\left(V_{s}, \omega_{s}\right)$, the object's location in the image $(u, v)$, and the optic flow $(\dot{u}, \dot{v})$. Note that only the optic flow due to translation $\left(\dot{u}_{t}, \dot{v}_{t}\right)$ is a function of the object's range; therefore, range can be estimated only when the camera is translating. Furthermore, the optic flow due to translation will be zero at an image location $\left(f V_{x s} / V_{z s}, f V_{y s} / V_{z s}\right)$ known as the focus of expansion (FOE). Since the FOE corresponds to the intersection of the velocity vector with the image plane, range estimates cannot be obtained for objects along the camera's instantaneous direction of motion. Additionally, the ability to estimate range deteriorates for objects whose translational optic flow is small, such as objects that are far away or that appear near the FOE.

Optic-flow information and ultimately a range estimate is associated with features in an image, where a feature is defined as a small region of interest within the image. The optic flow measurements are obtained from the difference between an object's location in successive images. The number of features for which range estimates can be obtained depends directly on the ability to select robust features that can be unambiguously tracked between images. The quality of the range estimate for each feature depends on the ability to accurately track the feature between images. In this implementation features are squares of $11 \times 11$ pixels which exhibit intensity variation greater than some user-specified minimum threshhold value. A correlation method is used to determine the feature's location in each new image.

Using the optic-flow equations (3), we formulate the computation of range as a state estimation problem using a Kalman filter. The Kalman filter is well-suited to this application because it combines redundant measurements to recursively improve its estimate over time. In addition, the state covariance matrix provided by the Kalman filter gives an indication of the estimate accuracy. The Kalman filter also yields a prediction of the state vector, the state covariance matrix, and an expected location of the feature for the next sample time. This latter information is used to constrain the search area for locating the feature in the next image. As the range information improves, the search window becomes smaller and less computation is required to locate the feature.

Several Kalman filter implementations were studied by Sridhar and Phatak [2], who obtained 
the best results by selecting the state vector $X=\rho_{s}$ and the measurement vector $Z=\{u, v\}$. With these definitions, the Coriolis equation (2) becomes the state equation and the perspective projection equations (1) become the measurement equations. The state and measurement equations can be written as follows

$$
\begin{aligned}
\dot{X} & =-\left[\omega_{s}\right] X-V_{s} \\
Z & =h(X)=\left[f x_{s} / z_{s}, f y_{s} / z_{s}\right]^{T}
\end{aligned}
$$

where

$$
\left[\omega_{s}\right]=\left[\begin{array}{ccc}
0 & -\omega_{z s} & \omega_{y s} \\
\omega_{z s} & 0 & -\omega_{x s} \\
-\omega_{y s} & \omega_{x s} & 0
\end{array}\right]
$$

The state equation is a time-varying linear system that depends on the camera's translational and rotational velocities. The measurement equation is a nonlinear function of the state.

The continuous-time state and measurement equations can be converted to their discrete-time equivalents assuming that $V_{s}$ and $\omega_{s}$ are constant during the sampling interval $\Delta T$. The discrete time system equations are

$$
\begin{aligned}
X(k+1)= & \Phi(k) X(k)+\Gamma(k) U(k) \\
& +\Gamma_{d}(k) \zeta_{x}(k) \\
Z(k)= & h[X(k)]+\zeta_{z}(k)
\end{aligned}
$$

where $\Phi(k)$ is the state transition matrix, $\Gamma(k)$ is the input distribution matrix, $U(k)=-V_{s}(k)$ is the control matrix, $\Gamma_{d}(k)$ is the disturbance distribution matrix, and $\zeta_{x}(k)$ and $\zeta_{z}(k)$ model the process noise and measurement noise, respectively. Zeromean Gaussian white noise is assumed such that $R(k) \equiv \operatorname{cov}\left(\zeta_{z}\right)$ and $Q(k) \equiv \operatorname{cov}\left(\zeta_{x}\right)$. The state transition matrix and the control distribution matrices have been derived by Sridhar and Phatak [2]. The measurement equation is linearized about the current estimate of $X$ giving

$$
\begin{aligned}
Z(k) & =H(k) X(k)+\zeta_{z}(k) \\
H(k) & =\partial h(X) / \partial X \\
& =f\left[\begin{array}{ccc}
1 / z_{s} & 0 & -x_{s} / z_{s}^{2} \\
0 & 1 / z_{s} & -y_{s} / z_{s}^{2}
\end{array}\right]_{\rho_{s}=\hat{\rho}_{s}(k-1)}(11)
\end{aligned}
$$

where $H(k)$ is computed based upon the best state estimate available just before the measurement update. The discrete-time state equation (8) and the linearized measurement equation (10) are used to recursively estimate the state vector $X$ and the state covariance matrix $P$.
The Kalman filter consists of two parts: the measurement update which improves the state estimate given a new measurement, and the time update which propagates the state forward in time according the the system dynamics. Before each iteration of the Kalman filter, we know $Q(k)$ and $R(k)$ and we have estimates of $X(k)$ and $P(k)$. The measurement update is then performed according to the following equations

$$
\begin{aligned}
\tilde{X}(k) & =\hat{X}(k)+K(k)[Z(k)-H(k) \hat{X}(k)] \\
\tilde{P}(k) & =[I-K(k) H(k)] P(k)
\end{aligned}
$$

where $H(k)$ is computed from $\hat{X}(k)$ as described above and the Kalman filter gain $K(k)$ is computed using the equation

$$
K(k)=P(k) H^{T}(k)\left[H(k) P(k) H^{T}(k)+R(k)\right]^{-1} .
$$

The time update equations are

$$
\begin{aligned}
\hat{X}(k+1)= & \Phi(k) \bar{X}(k)+\Gamma(k) U(k) \\
\hat{P}(k+1)= & \Phi(k) \tilde{P}(k) \Phi(k)^{T} \\
& +\Gamma_{d}(k) Q(k) \Gamma_{d}(k)^{T}
\end{aligned}
$$

As noted above, the Kalman filter requires initial estimates for $X$ and $P$. The initial estimate for $X$ can be derived from the optic flow equations and the perspective projection equations given a feature's location in two images and the camera's translational and rotational velocities which are assumed constant during the interval between images. First, the optic flow equations (3) are solved for $z_{s}$. The optic-flow equations actually comprise an overdetermined system of two equations in the one unknown $z_{s}$, so a single quadratic equation in $z_{s}$ is formed by summing the squares of the two optic-flow equations. Once $z_{s}$ is found, $x_{s}$ and $y_{s}$ can be determined from the perspective projection equations (1). The initial estimate of the state covariance matrix is chosen a priori.

A feature belonging to a far-away object or a feature near the FOE may have an inter-image motion smaller than can be resolved by the measurement process. The effective signal-to-noise ratio of shift measurements can be increased by skipping image frames to lengthen the time interval between images. Feature matching will then take place between images that are $n$ frames apart, where $n$ is the frame skipping factor. The Kalman-filter time updating is still performed at the original rate because the camera's motion information does not change. The measurement update is performed less frequently because each feature's location is measured only every 


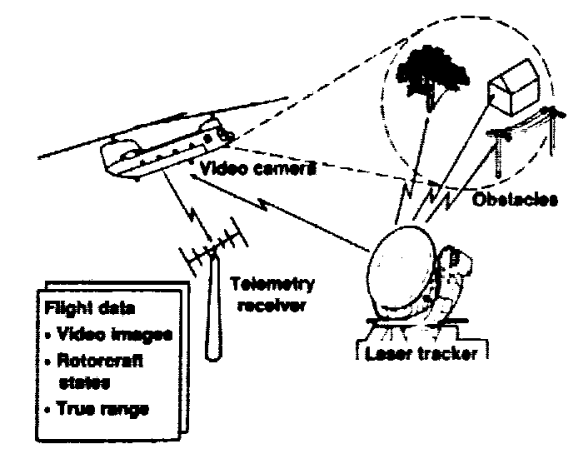

Figure 2: Flight Experiment Overview

$n$ frames. When the time index $k$ is a multiple of $n$, a full measurement update is performed according to equations $(12,13)$ above; otherwise, a trivial measurement update is performed according to the following equations

$$
\begin{aligned}
\tilde{X}(k) & =\hat{X}(k) \\
\tilde{P}(k) & =\hat{P}(k)
\end{aligned}
$$

Note that for a frame skipping factor of one every image is processed and the modified measurement update equations degenerate to the original form given in equations $(12,13)$. The effect of varying measurement update rate will be discussed in Section 4 where a reference image sequence will be subsampled using several values for the frame skipping factor.

\section{Experimental Flight Data}

In order to test the range estimation algorithm with actual data, a helicopter flight experiment was conducted at NASA Ames [6, 7]. The measurements included video imagery, the helicopter motion state, true range information, and the camera's characteristic parameters. The flight experiment is illustrated in Figure 2.

A monochrome video camera was mounted in the nose of a $\mathrm{CH}-47$ Chinook helicopter for image collection. The video signal was recorded onboard the helicopter with a U-matic video recorder and later digitized for computer processing. State measurements were acquired from an inertial navigation unit (INU) and discrete sensors such as accelerometers and rate gyros onboard the helicopter. The true range information was obtained using a groundbased laser tracker to measure the position of the helicopter-mounted camera and the (stationary) targets. Correlation between the airborne data (video imagery and state data) and the ground laser position data was accomplished using synchronized time sources. On the helicopter a time message was superimposed in a corner of the image and on the ground the measurements were stamped with the current time.

Knowledge of the camera's installation geometry and its imaging properties is also necessary to perform range estimation. It was assumed in the algorithm development that the camera's motion was available in the camera's axis system, and that an image location could be directly measured in terms of $u$ and $v$ as given in the perspective projection equations. In each case, the desired information must be derived from raw measurements and knowledge of the camera calibration parameters. The camera parameters consist of external parameters which describe the camera's geometric installation including its position and orientation with respect to the helicopter's axis system, and internal parameters which describe the imaging properties of the camera such as the lens focal length, the spacing of image pixels, and the pixel location where the $z_{s}$ axis intersects the image plane. The camera parameters were determined experimentally as described in an earlier paper [6].

During post-flight processing, the raw state measurements were filtered and checked for consistency. The measurements were sampled at $100 \mathrm{~Hz}$ and have a signal bandwidth of $10 \mathrm{~Hz}$. Measurements of velocity, although available on most operational helicopters, could not be obtained with the available equipment on the $\mathrm{CH}-47$ and had to be derived from the remaining state measurements (position, acceleration, orientation, and rotational velocity) using a state-estimation technique. Instrument bias and scale-factor errors were also identified and removed using state estimation. The resulting state information was converted to the camera's axis system and subsampled to video rate $(30 \mathrm{~Hz})$. The variables which describe the relation between the helicopter body axes and the camera axes are the external camera calibration parameters. The laser measurements of position were also corrected for the offset between the laser-track point and the camera's location. The difference between the target locations and the camera location gives the true range measurements (or more accurately, the true relative displacement vectors) which can be expressed in Earth, body, or camera axes.

The analog video signal recorded onboard the helicopter was digitized to produce $512 \times 512$ pixel 
images with 256 gray levels. Given the digitized images, an image plane location can be measured in terms of its row and column indices in the pixel array. An image plane location in $(u, v)$ coordinates must be derived using the following equations

$$
\begin{aligned}
& u=\left(n_{u}-n_{u_{o}}\right) \delta u_{e} \\
& v=\left(n_{v}-n_{v_{o}}\right) \delta v_{e}
\end{aligned}
$$

where $\left(n_{u}, n_{v}\right)$ is the grid location of the pixel, $\left(n_{u_{o}}, n_{v_{o}}\right)$ is the grid location where the $z_{s}$ axis passes through the image plane, and $\left(\delta u_{e}, \delta v_{e}\right)$ is the effective pixel size. The variables $n_{u_{o}}, n_{v_{o}}, \delta u_{e}, \delta v_{e}$, and the lens focal length $f$ are the internal camera calibration parameters.

The digitized images, camera state information, true range measurements, and camera calibration parameters resulting from a particular flight scenario have been collected into data sets consisting of a sequence of images with headers and a separate file containing the true target position data. The data sets for four flight scenarios have been collected into a database which may be requested from NASA Ames [7]. The four flight scenarios are described below:

1. Line Data Set: The helicopter is flying a straight line path between two rows of trucks parked on a runway in this scenario. The helicopter has an altitude of about $15 \mathrm{ft}$ above the runway and is flying at a ground speed of $35 \mathrm{ft} / \mathrm{sec}$. At this speed, the helicopter moves roughly $1 \mathrm{ft}$ between successive images. The image sequence consists of 240 frames (or $8 \mathrm{sec}$ onds of flight) during which time the helicopter travels approximately $290 \mathrm{ft}$. The location of two points on each truck has been measured to provide true range information. Range to the trucks varies between 200 and $800 \mathrm{ft}$ relative to the camera's initial position.

2. Arc Data Set: In this scenario the helicopter follows an S-shape ground track between the same arrangement of trucks described above. The helicopter alternates between fying toward the trucks on its left and those on its right. The helicopter maintains an altitude of $15 \mathrm{ft}$ and a speed of $40 \mathrm{ft} / \mathrm{sec}$. The peak yaw rate is approximately $0.13 \mathrm{rad} / \mathrm{sec}$. During 8 seconds of light (corresponding to a 240-frame image sequence) the helicopter moves $350 \mathrm{ft}$ toward the trucks. The visible trucks are 200 to $650 \mathrm{ft}$ away from the camera.
3. Posts Data Set: This scenario shows the helicopter flying a straight line path $35 \mathrm{ft}$ above a field containing a road, fence posts, and a building with a tower. The helicopter's speed is $40 \mathrm{ft} / \mathrm{sec}$. The image sequence contains 90 frames ( 3 seconds of flight) and the camera's travel is $120 \mathrm{ft}$. The true range has been measured for 13 objects varying between 80 and $350 \mathrm{ft}$ from the camera's initial position.

4. Towers Data Set: The helicopter flies toward several large power transmission towers against a background of hills while following a straight line flight path. The helicopter's speed is $90 \mathrm{ft} / \mathrm{sec}$ and the image sequence consists of 90 frames. True range data are not available for this image sequence.

The next section will present range results obtained with these four data sets using the algorithm discussed earlier.

\section{Range Estimation Results}

\subsection{Evaluation Method}

It is difficult to define a single measure for evaluating range estimation algorithms. Potentially, range accuracy can be evaluated on a pixel-by-pixel basis provided that sufficient truth data are available. In practice range estimates and truth data are sparse so that overall algorithm performance depends not only on range accuracy but also on the distribution of image features where range estimates are available. Because the distribution of image features resulting from different algorithms will vary, a more meaningful comparison is based on groups of features belonging to a single object. The resulting object range and size information is precisely the input required to plan an obstacle-free flight path. For this reason, the results presented here focus on comparison of (a) the range estimates of various objects, and (b) the range estimates of the individual features belonging to a single object. It is important to note that the algorithm described earlier automatically selects features and generates range estimates. Only when this process is complete are the resulting range estimates manually grouped for the purpose of performance evaluation. 

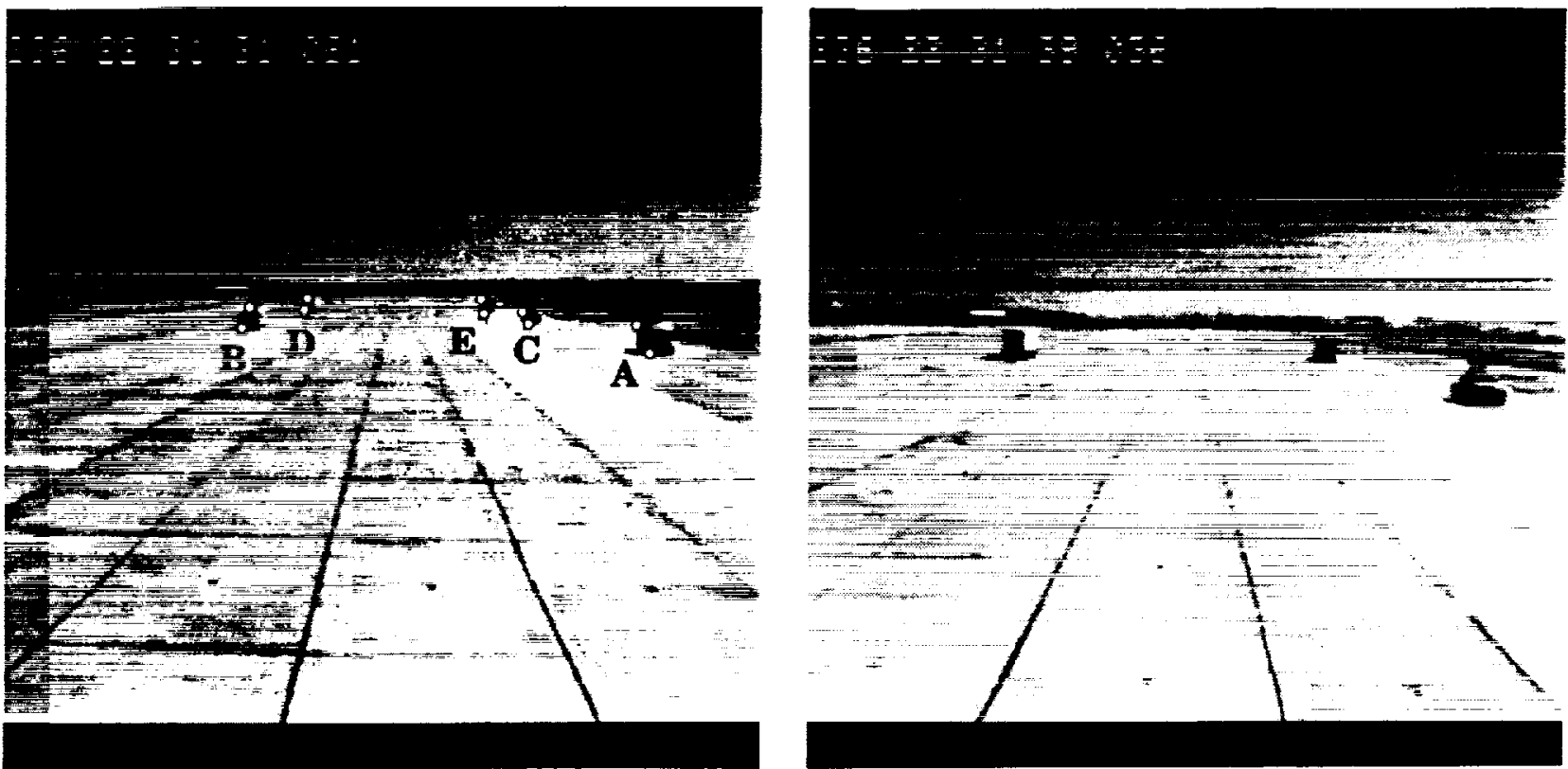

Figure 3: First and Last Images of the Line Sequence

The four data sets described in the previous section have been chosen to demonstrate maneuvers and/or obstacles encountered during lowaltitude helicopter flight. The Line sequence demonstrates the algorithm's performance during nominally straight and level flight conditions. The additive effect of curvilinear motion is provided in the Arc sequence. The Posts and Towers sequences contain obstacles likely to be encountered in lowaltitude flight. The results obtained with the Line sequence are described at length in the next section. The remaining sequences have been studied in similar detail, but for brevity only the unique aspects of each will be discussed.

\subsection{Line Data Set}

The first and last images of the 240-frame Line sequence taken during straight line flight are shown in Figure 3. The labeled points in the first image indicate locations where the true range has been measured. The camera's path and the measured truck locations are shown in Figure 4. The camera's measured velocity is shown in Figure 5. The helicopter's attitude changes continuously even during straight and level flight which manifests itself as considerable motion of the FOE. Using the measurements of $V_{s}$ and $\omega_{s}$, the FOE location has a range of travel of about 40 pixels both horizontally and vertically as shown in Figure 6.

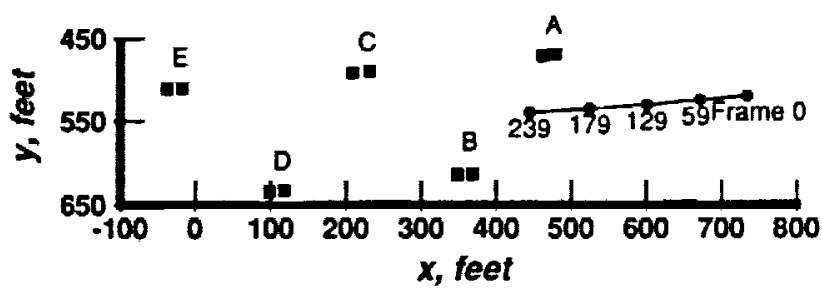

Figure 4: Camera Path and Truck Locations for Line Sequence

Range estimation was performed on this sequence with the parameters

$$
\begin{aligned}
& P(0)=\left[\begin{array}{ccc}
30^{2} & 0 & 0 \\
0 & 30^{2} & 0 \\
0 & 0 & 100^{2}
\end{array}\right] \mathrm{ft}^{2} \\
& R(k)=\left[\begin{array}{cc}
0.5 & 0 \\
0 & 0.5
\end{array}\right] \text { pixels }^{2} \\
& Q(k)=\left[\begin{array}{lll}
0 & 0 & 0 \\
0 & 0 & 0 \\
0 & 0 & 0
\end{array}\right]
\end{aligned}
$$

Figure 7 shows the image locations where range estimates were obtained in the 65th frame of the sequence. The FOE's location for this image is depicted by crosshairs in the figure. A total of approximately 250 range estimates were obtained in the image shown. The range estimates belonging to 


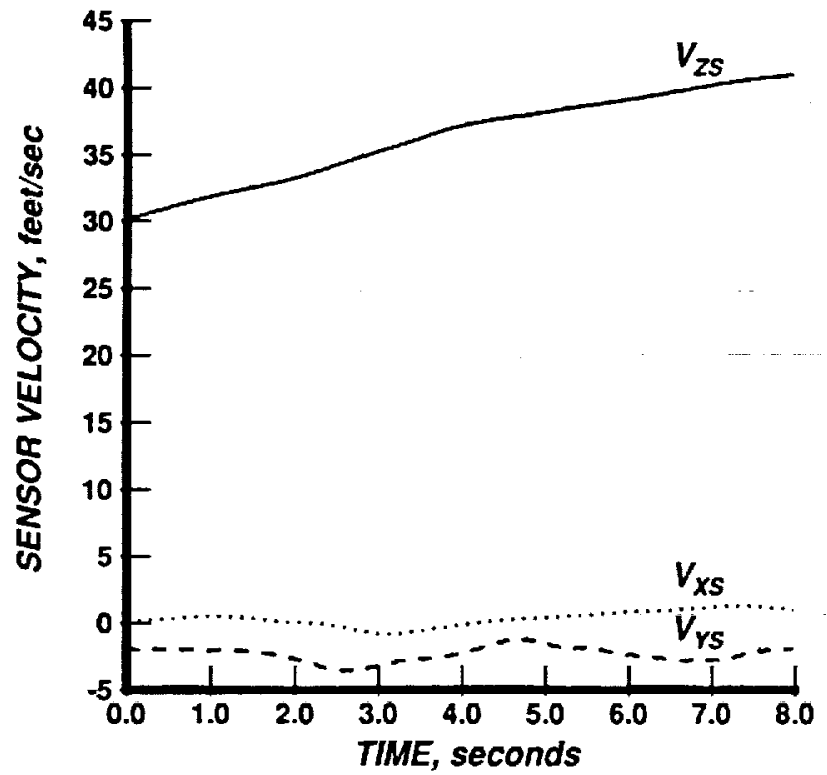

Figure 5: Camera Velocity for Line Sequence

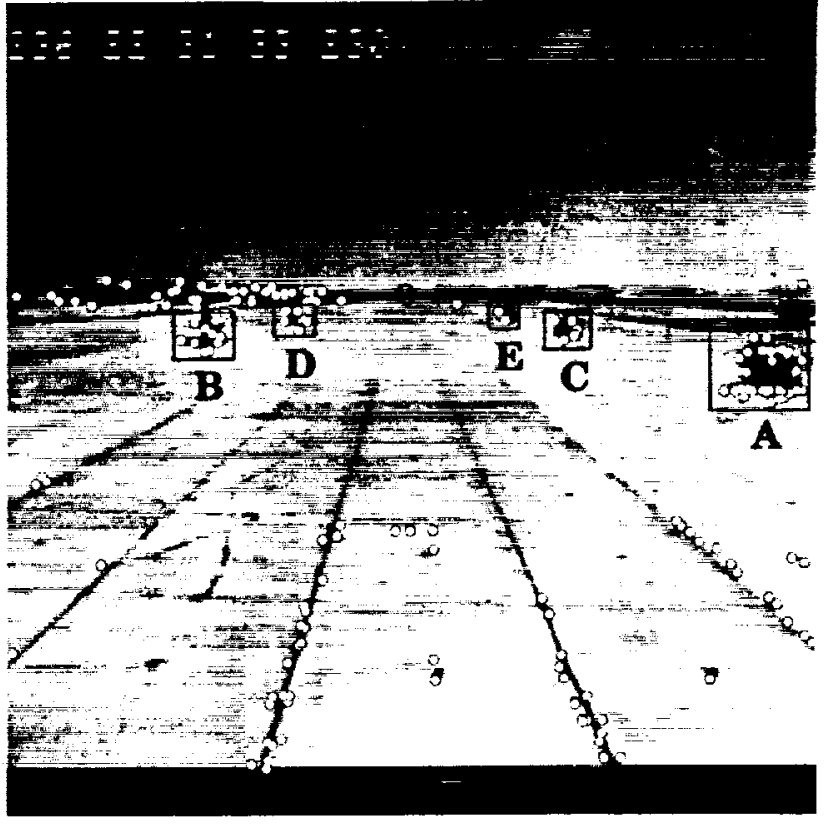

Figure 7: Range Estimates for Line Sequence (frame 65)

each truck are indicated by the boxes in Figure 7 . The range estimates for truck $A$ are plotted in Figure 8 along with the true range to the front and rear of the truck. Some of the range estimates start to die out near frame 70 as the truck begins moving out of the camera's field of view. The initial range estimates for new features may contain large errors because the optic flow is small and because the initial range estimate is based on only two mersurements. Truck A, for example, which is favorably. located near the camera and far from the FOE, his an inter-image shift of about 0.7 pixel relative to the FOE at the beginning of the sequence. In contrast. Truck $E$ is both far from the camera and close to the FOE, leading to an initial inter-image shift of only 0.1 pixel. During the same time, the FOE moves by approximately 1.5 pixels. The difficulty in measuring these small feature motions makes the process of range estimation for the flight data significantly more challenging and demonstrates the importance of subpixel measurement accuracy for this application. Figure 8 shows that even for poor initial range values, the Kalman filter will converge to a reasonable range estimate. The state covariance matrix provides an indication of the confidence associated with each estimate. The square root of the $z_{s}$ component of the state covariance matrix is shown in Figure 8 for the features belonging to truck $A$. 

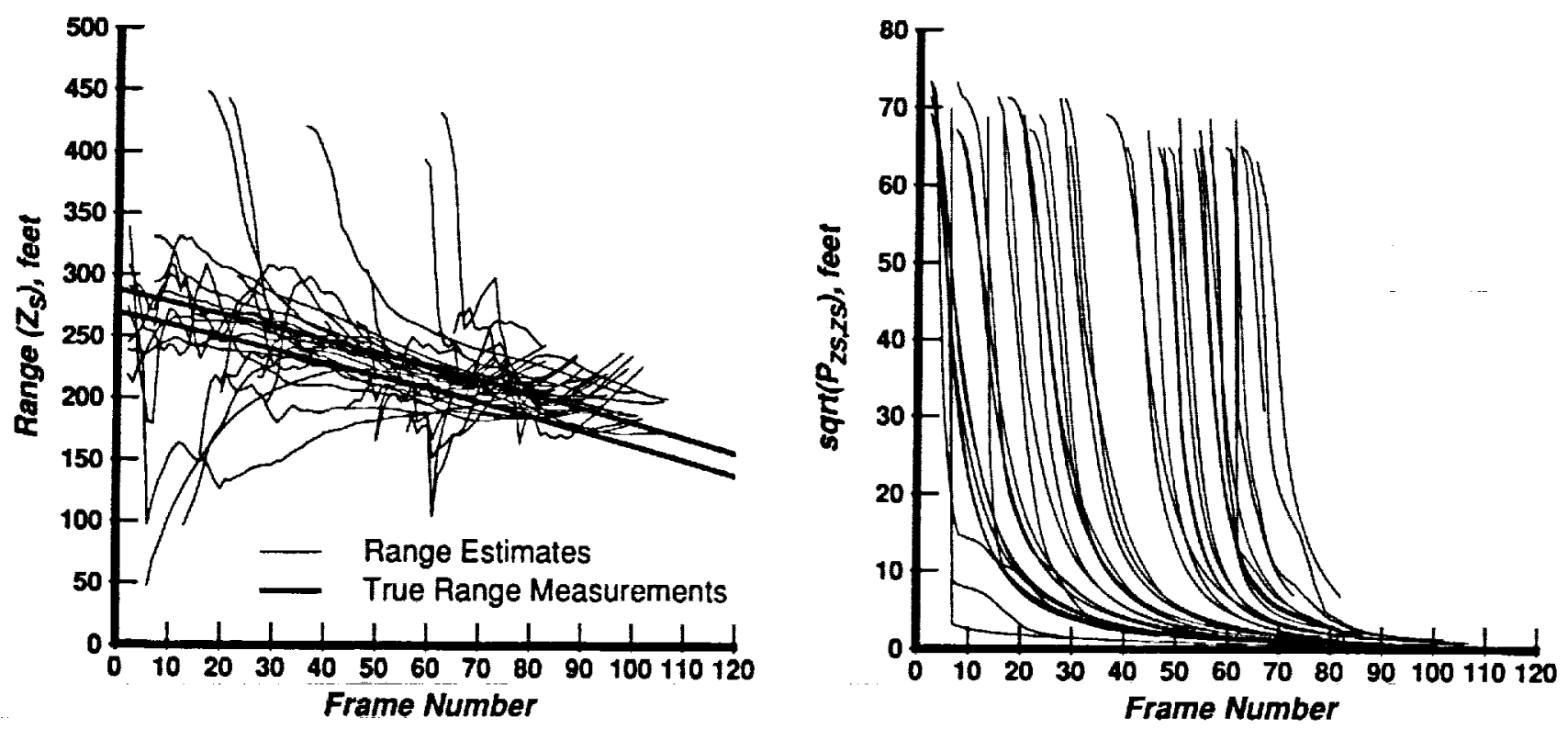

Figure 8: Range Estimates for Truck A in Line Sequence

The mean and standard deviation of the selected range estimates belonging to a truck can be compared with the true range measurements for that truck in order to evaluate the performance of the range estimation algorithm. An improved estimate of the object's range can be obtained by eliminating low-confidence range samples. Several criteria were used to identify undesirable range estimates including the following:

1. Percentage Method - Disregard the highest and lowest $20 \%$ of the range estimates.

2. Statistical Method - Compute the raw mean and standard deviation of the range samples. Disregard those range estimates lying more than 2 standard deviations from the mean.

3. Covariance Matrix Method - Use the state covariance matrix to disregard samples for which $\sqrt{P_{z s, z s}(i)}>\alpha \sqrt{P_{z s, z s}(0)}$. Values of $10 \%$ and $20 \%$ were used for $\alpha$.

The mean and standard deviations of the remaining samples give a composite range estimate for the truck. The resulting composite range estimates for truck $A$ are shown in Figure 9. Each of the trimming methods leads to a composite range estimate which lies between the front and back of the truck. The range estimates begin to diverge from the true range at frame 70 because the truck begins moving out of the camera's field of view. The features tracking the front of the truck die off first leaving a greater proportion of range estimates to the back of the truck, so the mean of the remaining estimates slowly increases. The standard deviation of the composite range estimate decays over time in a roughly exponential manner.

Similar range estimation results have been obtained for the remaining trucks as shown in Figure 10. The range estimates (trimmed using the Percentage Method) and true range measurements are tabulated in Table 1 for every 2 seconds of flight. The true range column gives the range to the midpoint of each truck while the true standard deviation represents half of the truck's length. As each truck is 20 feet in length, range errors less than about 10 feet are unimportant because they indicate range estimates which lie between the front and back of a truck. Table 1 shows that the range error and the standard deviation of the range estimate are greater for more-distant trucks. Both range errors and standard deviations decrease over time indicating improved accuracy and a greater uniformity of the individual estimates. The range estimates converge to within $10 \%$ of the true range for each truck. The standard deviation is less than half the truck length for the nearest three trucks.

The time required for the range estimation process to converge is also important in many applications. Table 2 shows the number of frames required for the range estimates to converge to within $10 \%$ 

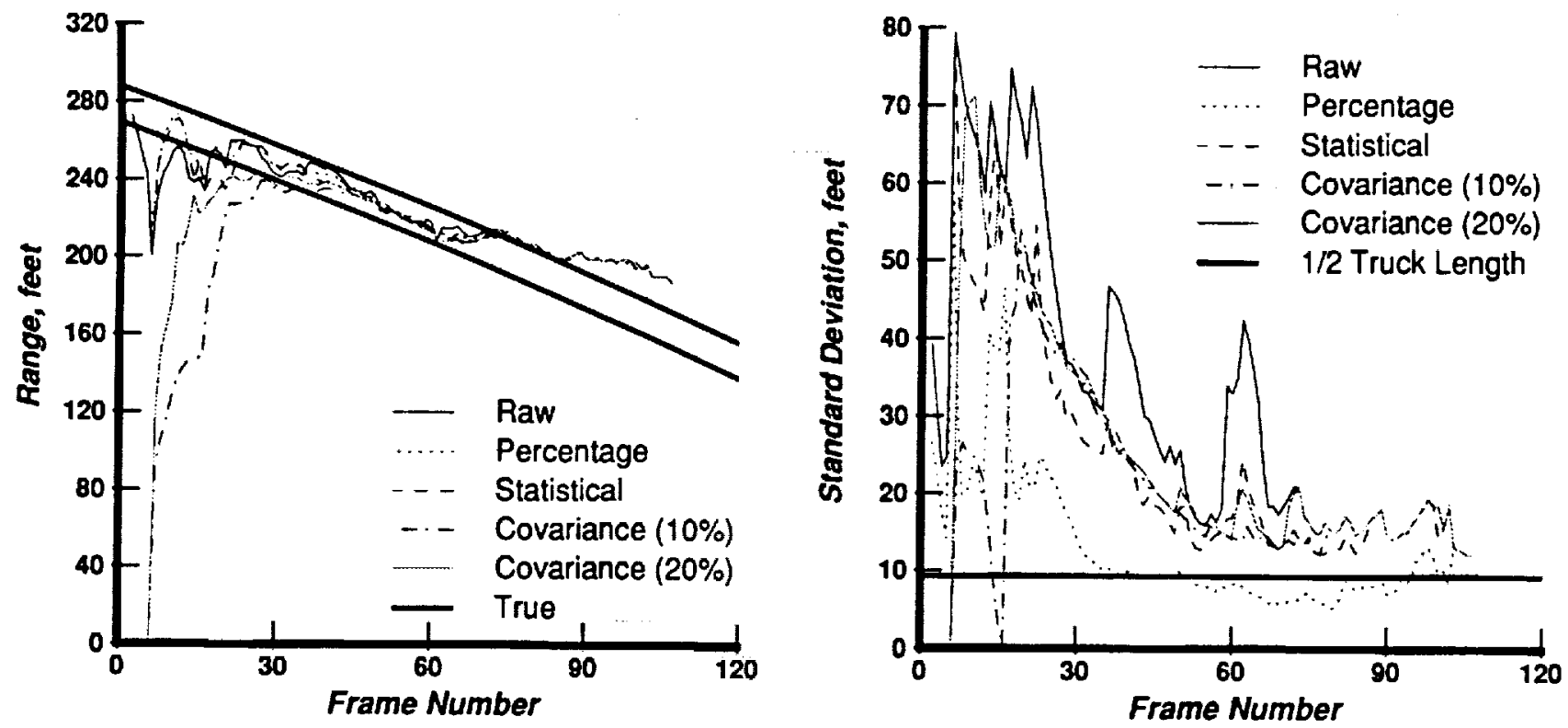

Figure 9: Composite Range Estimate for Truck A in Line Sequence

Table 1: Errors in Range Estimates of Trucks in Line Sequence

\begin{tabular}{|c|r|r|r|r|r|r|r|}
\hline Truck & Frame & $\begin{array}{c}\text { True } \\
\text { Range }\end{array}$ & $\begin{array}{c}\text { True } \\
\text { Std }\end{array}$ & $\begin{array}{c}\text { Est. } \\
\text { Mean }\end{array}$ & $\begin{array}{r}\text { Est. } \\
\text { Std }\end{array}$ & $\begin{array}{c}\text { Error } \\
\text { Range }\end{array}$ & $\begin{array}{c}\text { \% Error } \\
\text { Range }\end{array}$ \\
\hline \hline A & 59 & 217.9 & 9.2 & 216.1 & 7.8 & 1.8 & 0.83 \\
\hline B & 59 & 341.2 & 10.2 & 346.7 & 14.1 & 5.5 & 1.61 \\
B & 119 & 269.2 & 10.2 & 261.2 & 24.5 & 8.0 & 2.97 \\
B & 179 & 193.6 & 10.3 & 205.3 & 8.8 & 11.7 & 6.04 \\
\hline C & 59 & 468.4 & 11.2 & 412.0 & 50.4 & 56.4 & 12.04 \\
C & 119 & 398.6 & 11.3 & 386.7 & 20.6 & 11.9 & 2.99 \\
C & 179 & 321.1 & 11.3 & 312.2 & 14.1 & 8.9 & 2.77 \\
C & 239 & 239.5 & 11.3 & 232.5 & 6.8 & 7.0 & 2.92 \\
\hline D & 59 & 591.8 & 10.2 & 498.0 & 94.3 & 93.8 & 15.85 \\
D & 119 & 519.7 & 10.3 & 457.2 & 74.4 & 62.5 & 12.03 \\
D & 179 & 444.0 & 10.3 & 476.6 & 54.7 & 32.6 & 7.34 \\
D & 239 & 364.0 & 10.4 & 398.8 & 32.5 & 34.8 & 9.56 \\
\hline E & 59 & 716.7 & 10.3 & 577.5 & 85.7 & 139.2 & 19.42 \\
E & 119 & 646.8 & 10.3 & 620.9 & 79.3 & 25.9 & 4.00 \\
E & 179 & 569.1 & 10.4 & 525.4 & 81.6 & 43.7 & 7.68 \\
E & 239 & 487.2 & 10.4 & 482.9 & 52.7 & 4.3 & 0.88 \\
\hline
\end{tabular}



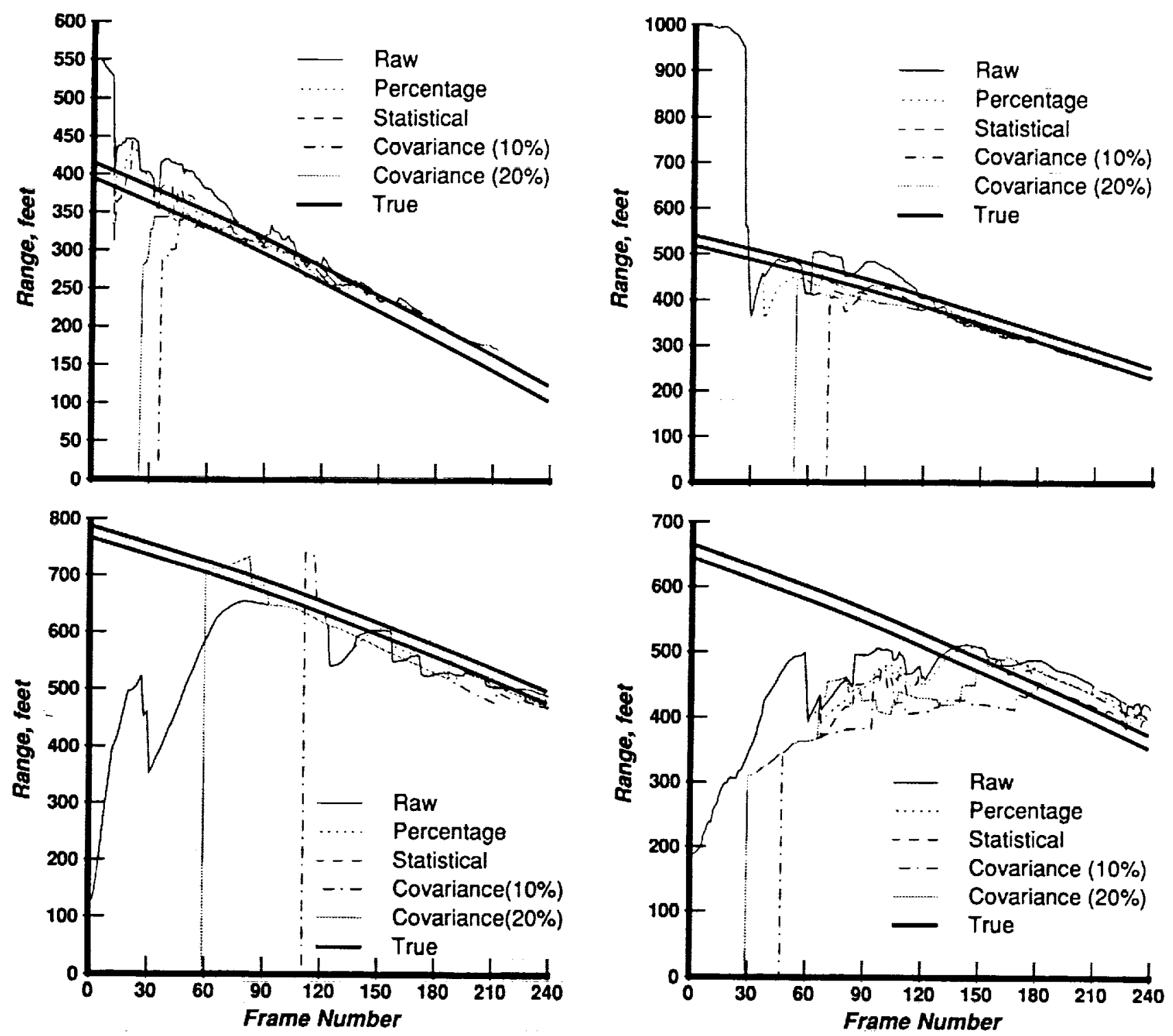

Figure 10: Composite Range Estimates for Trucks B-E in Line Sequence

Table 2: Convergence Time for Various Trimming Methods

\begin{tabular}{|c|c|c|c|c|c|c|c|c|c|c|}
\hline \multirow{3}{*}{ Truck } & \multicolumn{10}{|c|}{ Trimming Method } \\
\hline & \multicolumn{2}{|c|}{ Raw } & \multicolumn{2}{|c|}{ Percentage } & \multicolumn{2}{|c|}{ Statistical } & \multicolumn{2}{|c|}{ Cov $(10 \%)$} & \multicolumn{2}{|c|}{ Cov $(20 \%)$} \\
\hline & frame & range & frame & range & frame & range & frame & range & frame & range \\
\hline A & 9 & 269.9 & 7 & 271.9 & 7 & 271.9 & 26 & 252.7 & 19 & 259.9 \\
\hline B & 65 & 334.5 & 24 & 379.3 & 24 & 379.3 & 45 & 356.4 & 32 & 370.6 \\
\hline $\mathrm{C}$ & 35 & 493.7 & 47 & 481.1 & 35 & 493.7 & 71 & 455.3 & 54 & 473.7 \\
\hline D & 94 & 551.1 & 125 & 512.1 & 123 & 514.6 & 168 & 458.0 & 141 & 491.8 \\
\hline E & 69 & 705.9 & 69 & 705.9 & 69 & 705.9 & 118 & 648.1 & 60 & 715.6 \\
\hline
\end{tabular}


of the true mean range as a function of the various trimming methods. The Percentage Method and Statistical Method yield the best results in terms of minimizing convergence time. The time until convergence generally increases with range and proximity to the FOE. Truck D deviates from this trend, possibly due to tracking difficulties caused by distracting background objects (see Figure 7). Table 2 also gives each truck's true range at the time when convergence is achieved. The minimum distance at convergence is about $250 \mathrm{ft}$. Since the helicopter moves at about $35 \mathrm{ft} / \mathrm{sec}$, this would give a pilot a minimum of about 7 seconds of warning to avoid each of the trucks. In most cases, for a given distance of camera travel the algorithms have demonstrated the ability to estimate the range to objects up to 10 times farther away to within $10 \%$ error.

Inter-image feature shifts may be much less than a pixel as noted earlier. This is a difficulty since the ability to measure subpixel image motion is limited. The effective signal-to-noise ratio of shift measurements can be increased by skipping image $\bar{s}$ of the original sequence. Range estimation was performed with skipping factors of $1,2,3,4,5$, and 10 . The resulting range estimates were trimmed using the Percentage Method and the time to $10 \%$ convergence was determined. The convergence times given in Table 3 are expressed as the equivalent frame number of the original sequence so that the results of different skipping factors can be compared on an equal footing. The columns of Table 3 show that for a given frame skipping factor, convergence time increases with range. The rows of Table 3 show that for each truck there is some optimal frame skipping factor, say $n^{*}$, which yields a minimum convergence time (indicated by boxed entries in the table). Since Truck A exhibits little variation in convergence time, $n^{*}$ is chosen to be one. Truck $E$ has two local minima, both indicated by boxed entries. For frame skipping factors less than $n$ the convergence time increases due to the limited accuracy of feature shift measurements, while for frame skipping factors greater than $n^{*}$ the convergence time increases due to the decreased measurement rate. Finally, Table 3 shows the trend that the optimal frame skipping factor for minimum convergence time increases with an object's range.

\subsection{Arc Data Set}

The first and last images of the 240-frame Arc sequence taken during maneuvering flight are shown in
Table 3: Convergence Time for Various Frame Skipping Factors

\begin{tabular}{|c|rrrrrr|}
\hline Truck & \multicolumn{7}{|c|}{ Frame Skipping Factor } \\
& $\mathrm{n}=1$ & 2 & 3 & 4 & \multicolumn{1}{c|}{5} & 10 \\
\hline \hline A & 7 & 8 & 6 & 8 & 10 & 20 \\
B & 24 & 4 & 6 & 8 & 20 & 30 \\
C & 47 & 46 & 24 & 52 & 85 & 60 \\
D & 125 & 132 & 87 & 40 & 135 & 100 \\
E & 69 & 154 & 126 & 88 & 100 & 170 \\
\hline
\end{tabular}

Figure 11. The labeled points in the first image indicate locations where the true range has been measured. The camera's path and the measured truck locations are shown in Figure 12. The camera's measured linear and angular velocities are shown in Figure 13. The FOE travels about 150 pixels horizontally and 40 pixels vertically as shown in Figure 14.

The resulting range estimates (trimmed with the Percentage Method) are shown in Table 4. Truck D moves out of the field of view at frame 105 and re-enters the image at frame 190 . The range estimate for Truck $D$ (using the Percentage Method) is plotted in Figure 15. The range estimate for each truck is seen to converge to within about $10 \%$ of the true range. The number of frames required for convergence is given in Ta'le 5 along with the true range when convergence is achieved. The minimum range at convergence is for Truck B at $229.5 \mathrm{ft}$ which gives the pilot about 5.7 seconds of warning to react and plan a new path. Table 5 also gives the distance traveled by the helicopter before convergence is reached and the percentage of the initial obstacle range represented by the travel distance. The distance traveled for Truck B is $13.7 \%$ of its initial range, roughly the same fraction as observed for the Line sequence. This is not unexpected because over the first 27 frames of the sequence when the range estimate is converging, the helicopter's path is well approximated by a straight line (see Figure 12). The percentage distance traveled for Trucks $C$ and $D$ is probably a better indicator of the performance that can be expected during maneuvering flight. The convergence time for Truck $\mathrm{E}$ is significantly greater than for the other trucks although the range at convergence is comparable. The slower convergence for Truck $E$ is attributed to its proximity to the FOE as the FOE sweeps across the image. Figure 16 shows the distance between the FOE and each of the two 

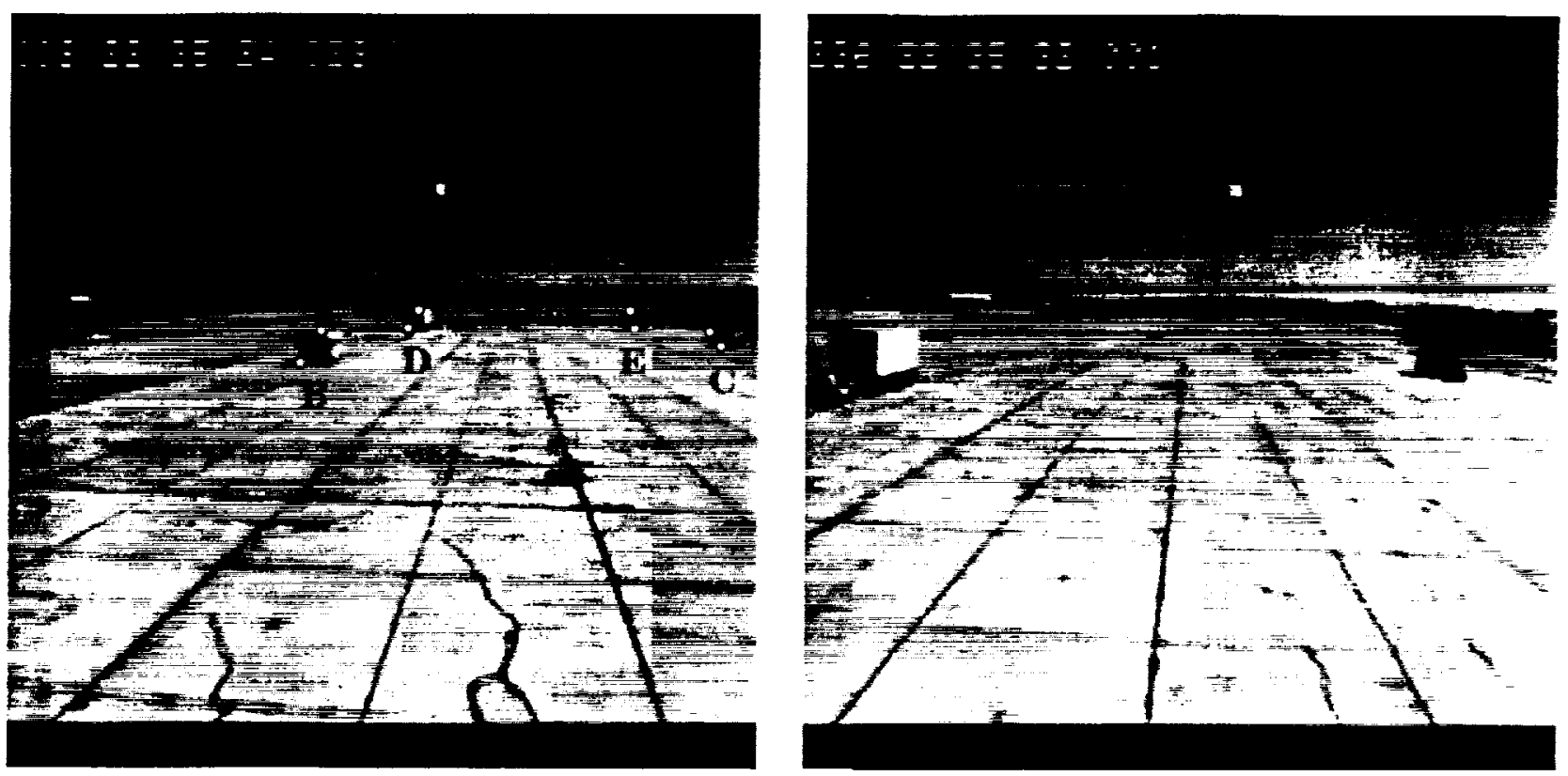

Figure 11: First and Last Images of the Arc Sequence

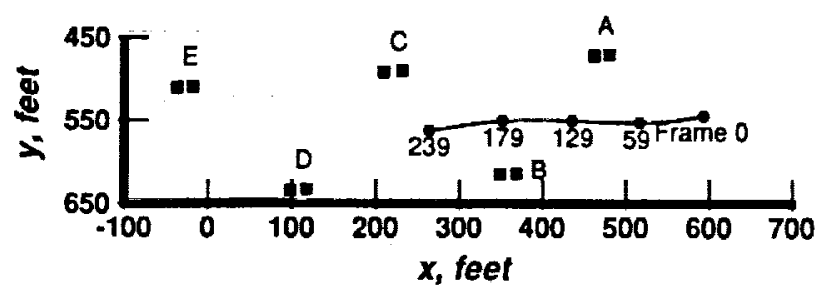

Figure 12: Camera Path and Truck Locations for Arc Sequence

known locations on Truck $\mathrm{E}$ as a function of the frame number.

The convergence time of the range estimates during maneuvering flight is greater than for straight line flight. The Kalman-filter-based range estimation procedure is expected to perform equally well under both flight scenarios and does not provide an adequate explanation for the performance degradation. More probably, the performance difference is due to errors in the motion data used by the Kalman filter. The helicopter's heading angle has the lowest resolution ( 0.7 degree) of the angular measurements collected during flight test. Normally, the helicopter's heading angle would not enter into the motion data used for range estimation if the linear velocity and angular velocity were directly measured in the helicopter's body axis system or in the camera's axis system. Recall, however, that linear velocity could not be directly measured during flight and instead had to be reconstructed using state estimation. The resulting velocity profile depends most heavily on the available position and acceleration measurements. The helicopter's heading angle plays an important role in transforming the position information measured in Earth axes into the helicopter's body axis system. An error of 0.7 degree in the helicopter's heading angle leads to an 8-pixel error in computing the FOE location. In practice, since the estimated velocity profile is a compromise between position and acceleration information, FOE location errors less than 8 pixels are expected. For straight and level flight the heading angle is slowly changing and the heading angle measurement error can be identified as a pure bias based on consistency between the position and acceleration measurements. The resulting FOE location errors are therefore minimal for straight line flight. During maneuvering flight, however, the heading-angle measurement error is only partially accounted for by bias correction. The remaining heading-angle measurement error translates directly into uncertainty in the FOE location which introduces errors into the range estimation process. In future flight experiments, this difficulty will be corrected by obtaining direct linear-velocity measurements or by obtaining heading-angle measurements of higher accuracy. 

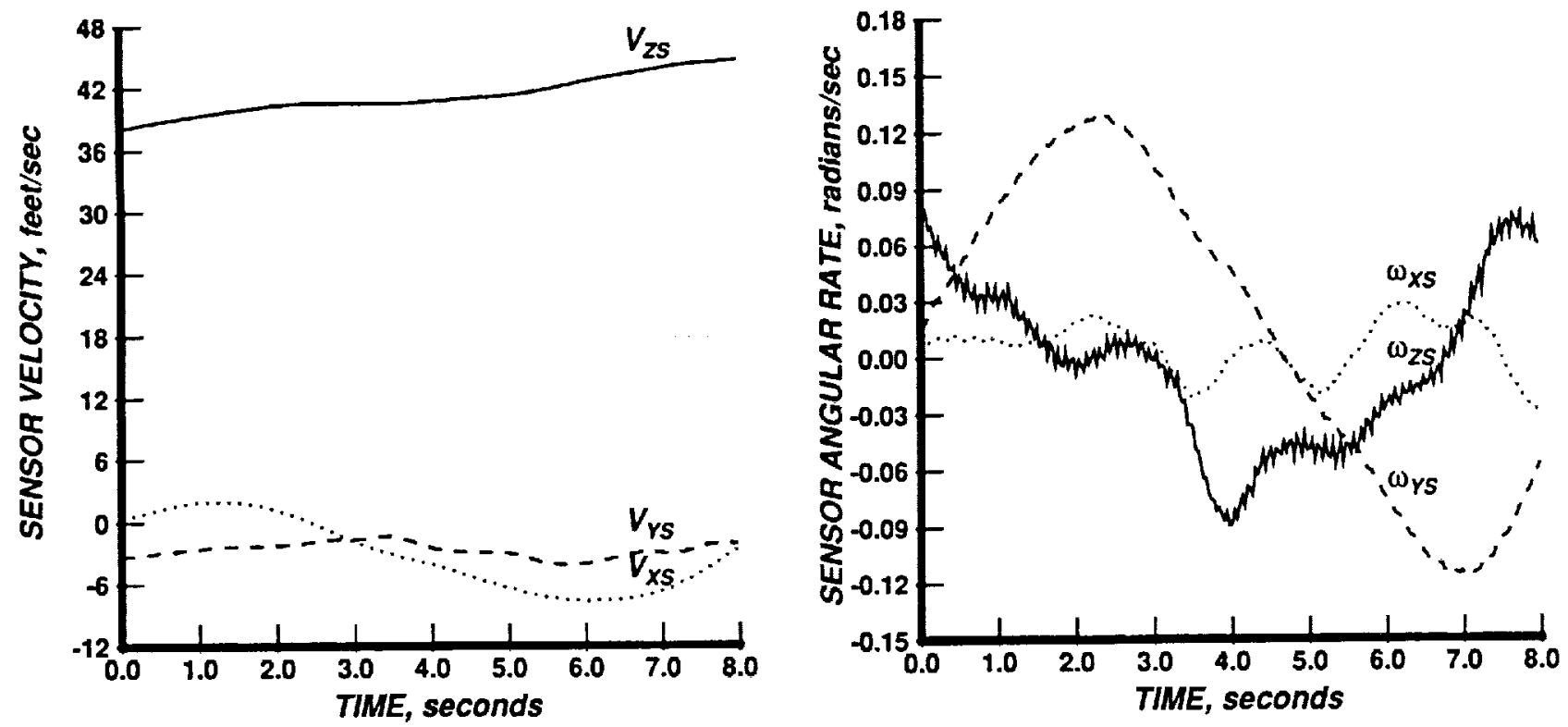

Figure 13: Camera Velocity for Arc Sequence

Table 4: Errors in Range Estimates of Trucks in Arc Sequence

\begin{tabular}{|c|c|c|c|c|c|c|c|}
\hline Truck & Frame & $\begin{array}{c}\text { True } \\
\text { Range }\end{array}$ & $\begin{array}{c}\text { True } \\
\text { Std }\end{array}$ & $\begin{array}{c}\text { Est. } \\
\text { Mean }\end{array}$ & $\begin{array}{c}\text { Est. } \\
\text { Std }\end{array}$ & $\begin{array}{c}\text { Error } \\
\text { Range }\end{array}$ & $\begin{array}{c}\text { \% Error } \\
\text { Range }\end{array}$ \\
\hline \hline B & 59 & 182.2 & 10.3 & 173.2 & 11.4 & 9.0 & 4.94 \\
\hline C & 59 & 313.8 & 11.3 & 373.8 & 25.3 & 60.0 & 19.12 \\
C & 119 & 242.6 & 11.0 & 261.5 & 20.2 & 18.9 & 7.79 \\
C & 179 & 158.1 & 11.1 & 142.0 & 5.0 & 16.1 & 10.18 \\
\hline D & 59 & 432.4 & 10.3 & 280.1 & 68.3 & 152.3 & 35.22 \\
D & 105 & 348.4 & 10.1 & 354.1 & 53.5 & 5.7 & 1.64 \\
D & 239 & 182.9 & 10.3 & 186.8 & 16.3 & 3.9 & 2.13 \\
\hline E & 59 & 561.7 & 10.4 & 207.8 & 0.0 & 353.9 & 63.01 \\
E & 119 & 484.6 & 10.2 & 333.2 & 45.3 & 151.4 & 31.24 \\
E & 179 & 402.4 & 10.2 & 316.1 & 42.6 & 86.3 & 21.45 \\
E & 239 & 306.6 & 10.3 & 275.6 & 32.6 & 31.0 & 10.11 \\
\hline
\end{tabular}

Table 5: Converge Time for Arc Sequence

\begin{tabular}{|c|c|c|c|c|c|}
\hline Truck & $\begin{array}{c}\text { Convergence } \\
\text { Frame }\end{array}$ & $\begin{array}{c}\text { True Range at } \\
\text { Convergence }\end{array}$ & $\begin{array}{c}\text { Initial } \\
\text { Range }\end{array}$ & $\begin{array}{c}\text { Distance } \\
\text { Traveled }\end{array}$ & $\begin{array}{c}\text { \% of Initial } \\
\text { Range Traveled }\end{array}$ \\
\hline \hline B & 27 & 229.5 & 265.8 & 36.3 & 13.7 \\
C & 80 & 290.7 & 375.5 & 84.8 & 22.6 \\
D & 92 & 376.0 & 514.2 & 138.2 & 26.9 \\
E & 239 & 306.6 & 621.7 & 315.1 & 50.7 \\
\hline
\end{tabular}


Table 6: Errors in Range Estimates of Objects in Posts Sequence

\begin{tabular}{|c|c|c|c|c|c|c|c|}
\hline Truck & Frame & $\begin{array}{c}\text { True } \\
\text { Range }\end{array}$ & $\begin{array}{c}\text { True } \\
\text { Std }\end{array}$ & $\begin{array}{c}\text { Est. } \\
\text { Mean }\end{array}$ & $\begin{array}{c}\text { Est. } \\
\text { Std }\end{array}$ & $\begin{array}{c}\text { Error } \\
\text { Range }\end{array}$ & $\begin{array}{c}\text { \% Error } \\
\text { Range }\end{array}$ \\
\hline \hline A & 35 & 129.80 & 0.00 & 137.20 & 0.90 & 7.40 & 5.70 \\
B & 55 & 130.60 & 0.00 & 134.80 & 0.30 & 4.20 & 3.22 \\
C & 89 & 137.10 & 0.00 & 142.80 & 4.00 & 5.70 & 4.16 \\
D & 89 & 194.10 & 0.00 & 192.30 & 0.00 & 1.80 & 0.93 \\
E & 89 & 301.30 & 9.40 & 302.70 & 7.30 & 1.40 & 0.46 \\
F & 89 & 304.30 & 0.50 & 313.50 & 5.00 & 9.20 & 3.02 \\
G & 89 & 401.50 & 4.40 & 354.20 & 19.60 & 47.30 & 11.78 \\
H & 89 & 192.10 & 0.00 & 268.80 & 0.00 & 76.70 & 39.93 \\
I & 89 & 129.50 & 0.00 & 130.50 & 5.30 & 1.00 & 0.77 \\
J & 89 & 87.40 & 0.00 & 92.80 & 1.50 & 5.40 & 6.18 \\
K & 70 & 99.40 & 0.00 & 103.50 & 2.70 & 4.10 & 4.12 \\
L & 70 & 97.50 & 0.00 & 95.30 & 2.40 & 2.20 & 2.26 \\
M & 55 & 93.30 & 0.00 & 91.50 & 2.00 & 1.80 & 1.93 \\
\hline
\end{tabular}

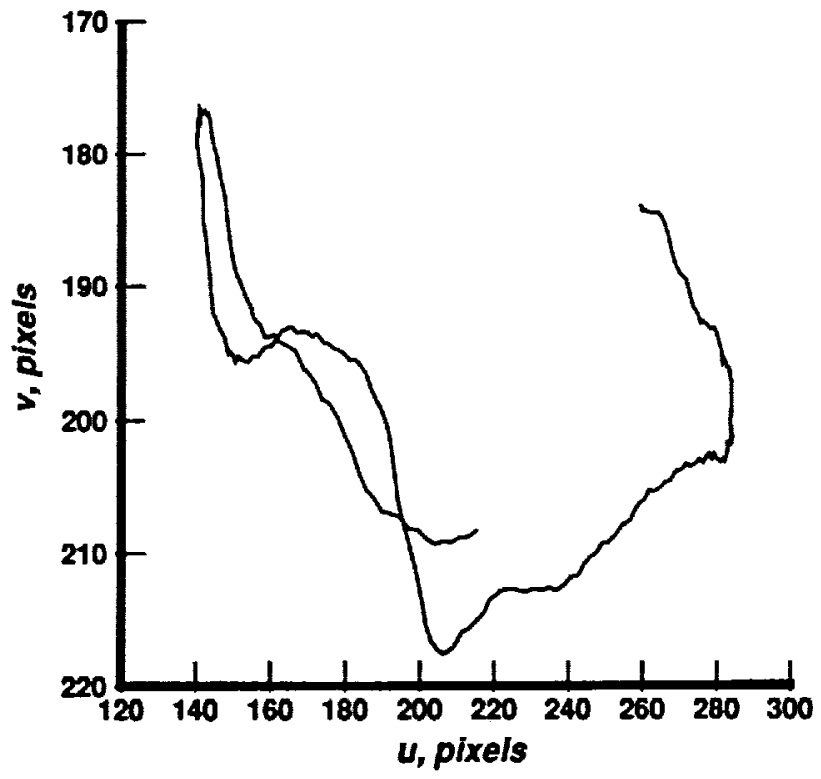

Figure 14: FOE Motion for Arc Sequence

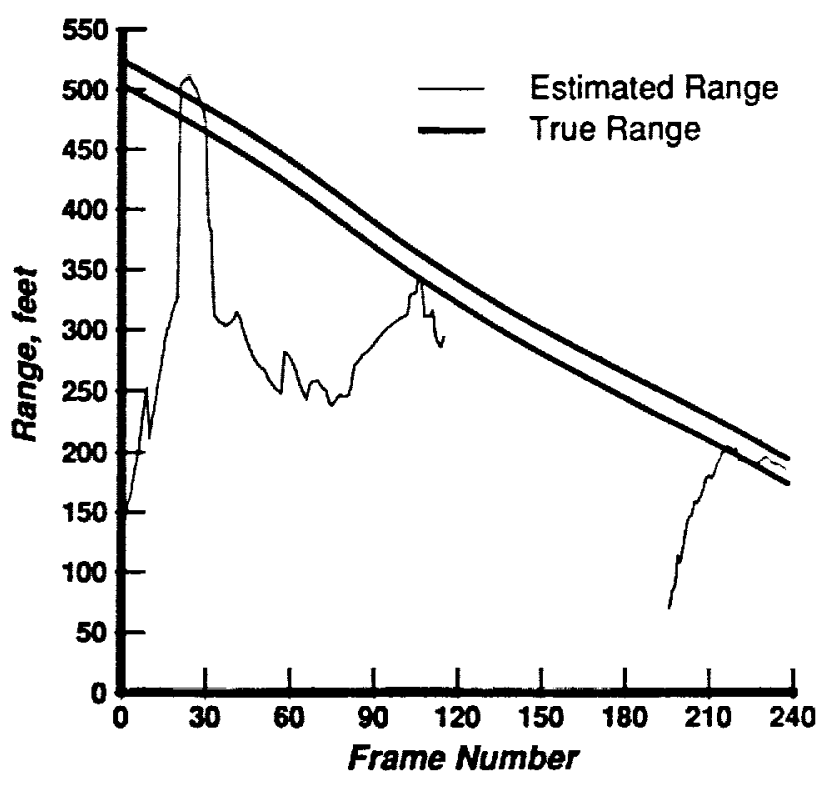

Figure 15: Composite Range Estimate for Truck D in Arc Sequence 


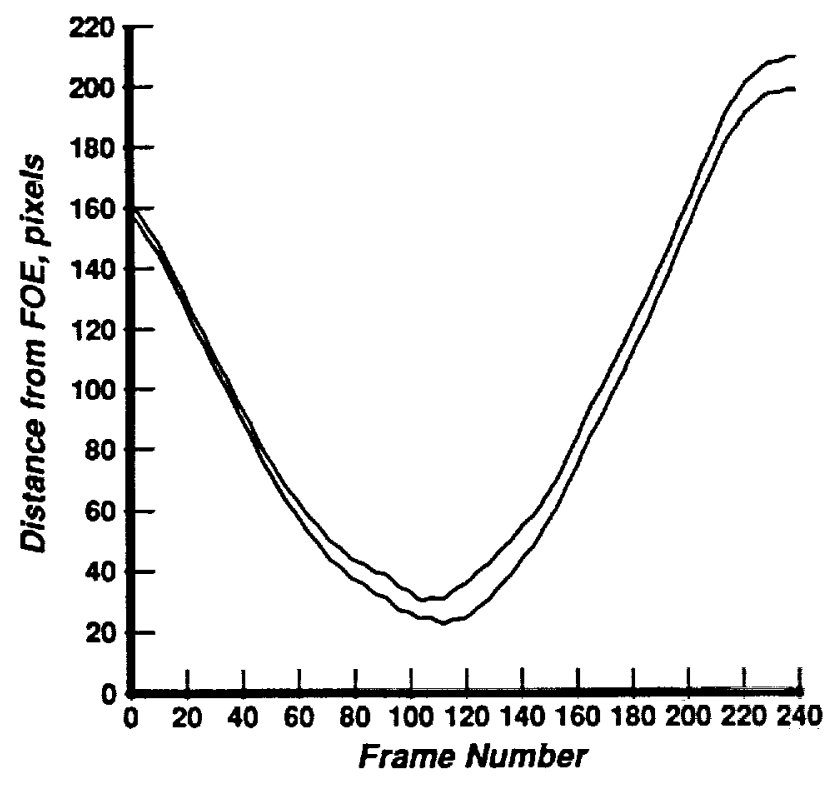

Figure 16: Proximity of Truck E to the FOE for Arc Sequence

\subsection{Posts and Towers Data Sets}

The first and last images of the 90-frame Posts sequence taken during 3 seconds of straight-line flight over a field with a road, a building, and several white posts are shown in Figure 17. The labeled points in the first image indicate locations where the true range has been measured. The sensor velocity is approximately $40 \mathrm{ft} / \mathrm{sec}$ during the sequence. The range estimates and true range measurements are shown in Table 6 for each of the objects labeled in Figure 17. The range estimate for an object corresponds to the last image frame in which the object appears. The range estimates are within $10 \%$ of the true value for all of the objects except $G$ and $H$. Due to its proximity to the FOE, the range estimate for object $G$ requires greater time to converge. Post $\mathrm{H}$ was first detected in frame 84 and requires more than the remaining 5 frames to converge.

Figure 18 shows the first and last images of the 90 -frame Towers sequence taken during 3 seconds of straight-line flight over power transmission towers. The sensor velocity is approximately $90 \mathrm{ft} / \mathrm{sec}$ during the sequence. True range measurements are not available for this sequence, so the range estimates can only be compared for consistency among the three towers indicated in Figure 18. The range estimates are 456.0, 442.0, and 441.1 feet for towers $A, B$, and C, respectively. The standard deviation of the estimates are 17.7, 15.4, and 15.6 feet. 

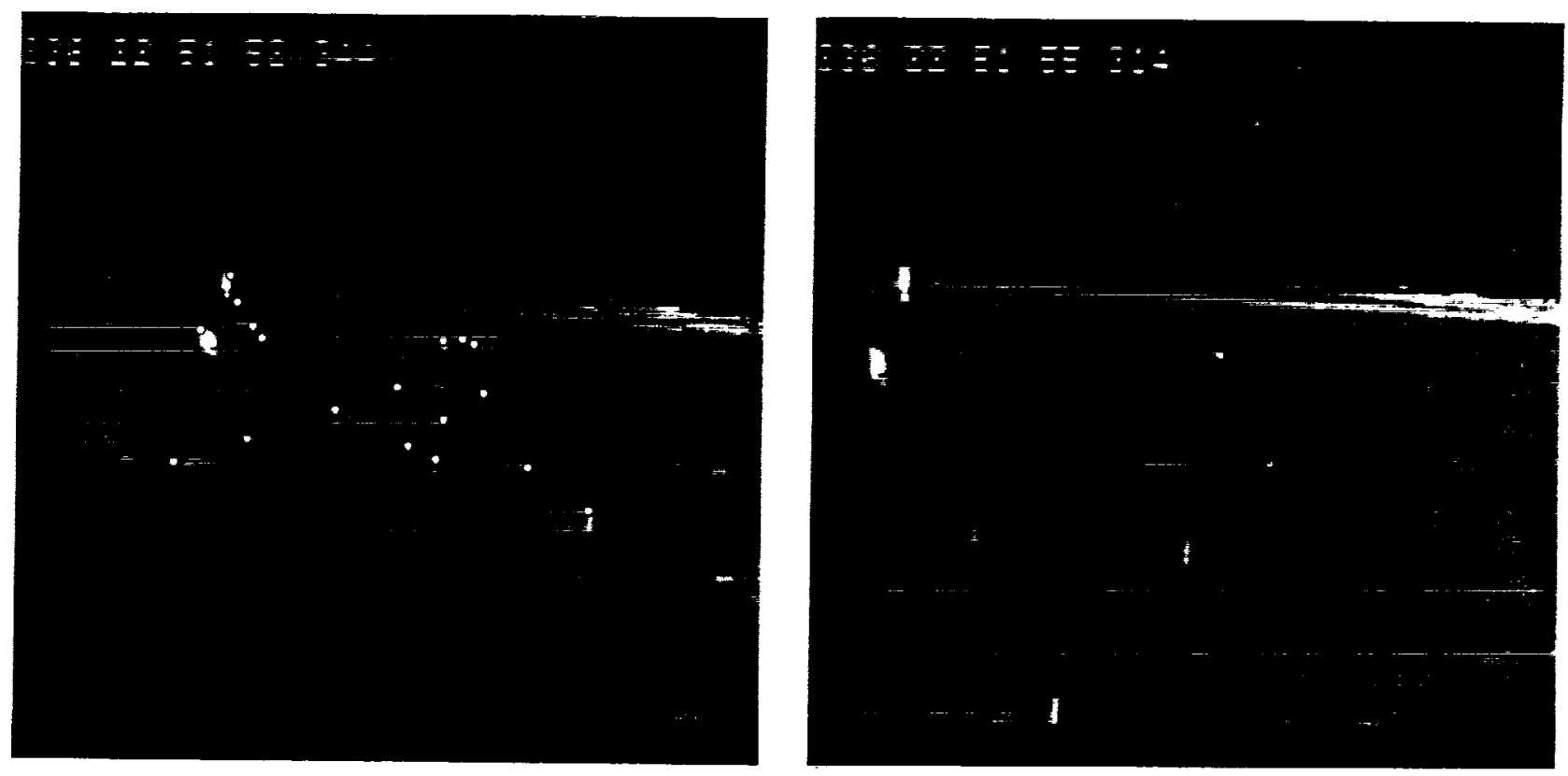

Figure 17: First and Last Images of the Posts Sequence
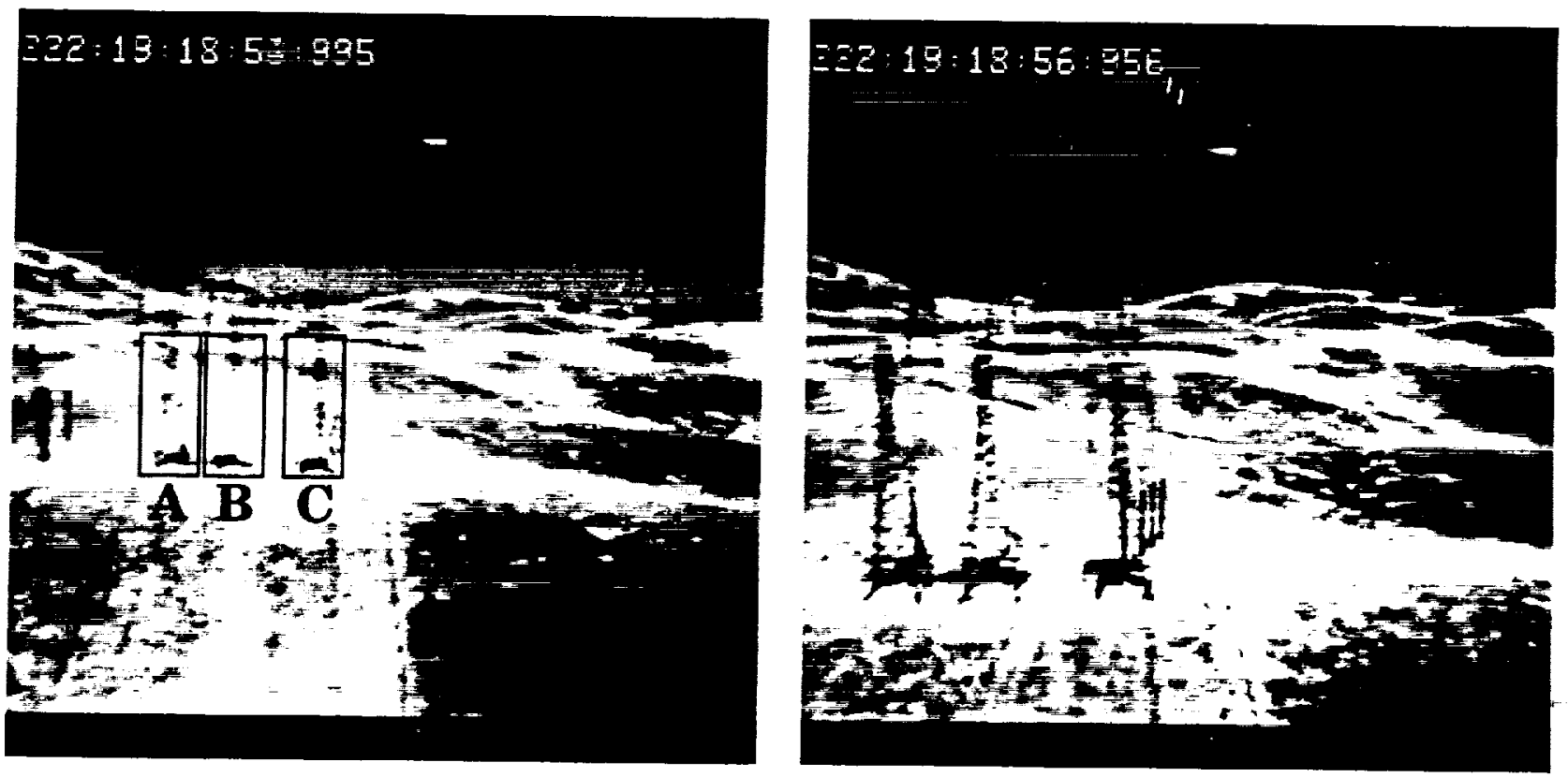

Figure 18: First and Last Images of the Towers Sequence 


\section{Conclusions}

In this paper actual flight data have been used to demonstrate the feasibility of passive range estimation from a helicopter equipped with a single camera and an inertial navigation system. Range estimation results were compared with true range measurements which were independently acquired. A feature-based method extracts optic-flow measurements from the image sequence. Then an extended Kalman filter uses the measurements of optic flow and camera motion to recursively estimate range. Range estimation results have been shown for straight-line and maneuvering flight paths over man-made and natural terrain.

The range estimation method yields results which compare closely with true range measurements. For straight-line motion, range estimates converging to within $10 \%$ of the true range by the time the helicopter has traversed $1 / 10$ th the distance to the object. For straight-line flight at $35 \mathrm{ft} / \mathrm{sec}$, obstacle ranges were estimated within $10 \%$ error at a minimum distance of $250 \mathrm{ft}$, giving the pilot approximately 7 seconds of warning to avoid the obstacles. During maneuvering flight the range estimates converged more slowly due to the relatively low resolution of angular motion information. For turning maneuvers at $40 \mathrm{ft} / \mathrm{sec}$, obstacle ranges were estimated within $10 \%$ error at a minimum distance of $230 \mathrm{ft}$, giving the pilot approximately 5.7 seconds of warning to avoid the obstacles.

The inter-image motion of distant objects encountered in the flight data was often significantly less than one pixel. For these objects, improved range accuracy and decreased convergence time are obtained by skipping images to effectively decrease the image sampling rate. In general, a particular value of the frame skipping factor leads to a minimum convergence time and this optimal frame skipping factor is seen to increase with the obstacle's range. An event-driven Kalman filter is currently being explored as one method for simultaneously treating quickly and slowly moving features. Here the Kalman filter time update occurs with every image as before, but the measurement update takes place only when the feature has moved some minimum distance (say, one pixel) from its previous measurement-update location.

The motion-based range estimation algorithm described here suffers difficulties near the FOE as do most motion-based methods. An event-driven Kalman filter may help to minimize this problem by improving range estimates to slowly-moving features. In another approach, a combined stereo and motion range estimation algorithm has been developed at Ames Research Center and tested with laboratory data [8]. A helicopter flight test program is under way to provide stereo images and motion measurements for use with this class of algorithms.

\section{References}

[1] V. H. L. Cheng, and Sridhar, B., "Considerations for Automated Nap-of-the-Earth Rotorcraft Flight," Journal of the American Helicopter Society, Vol. 36, No. 2, pp. 61-69, April 1991; also, Proceedings of the 1988 American Control Conference, Atlanta, GA, June 15-17, 1988.

[2] Sridhar, B., and Phatak, A., "Simulation and Anaysis of Image-Based Navigation system for Rotorcraft Low-Altitude Flight," American $\mathrm{He}$ licopter Society Specialists' Meeting on Automation Applications of Rotorcraft, Atlanta, GA, April 1988. To appear in IEEE Transactions on Systems, Man and Cybernetics.

[3] Sridhar, B., Suorsa, R., and Hussien, B., "Passive Range Estimation for Rotorcraft Lowaltitude Flight," NASA TM 103897, October 1990.

[4] Menon, P. K. A., and Sridhar, B., "Passive Navigation Using Image Irradiance Tracking," AIAA Guidance, Navigation, and Control Conference, Boston, MA, August 1989.

[5] Barniv, Y., "Velocity Filtering Applied to Optical Flow Calculations," NASA TM 102802, August 1990.

[6] Smith, P. N., "Flight Data Acquisition for Validation of Passive Ranging Algorithms for Obstacle Avoidance," NASA TM 102809, October 1990.

[7] Smith, P. N., "A Rotorcraft Flight Database for Validation of Vision-Based Ranging Algorithms," NASA TM 103906, April 1992.

[8] Sridhar, B., and Suorsa, R., "Integration of Motion and Stereo Sensors in Passive Ranging Systems," IEEE Transactions on Aerospace and Electronic Systems, Vol. 27, No. 4, pp. 741-746, July 1991. 


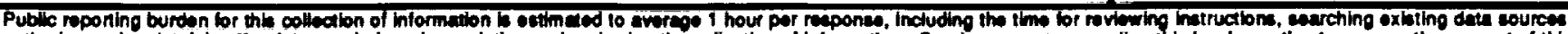

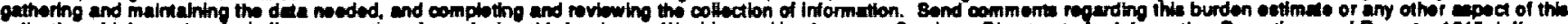

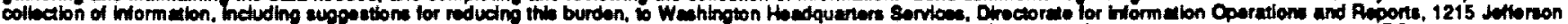

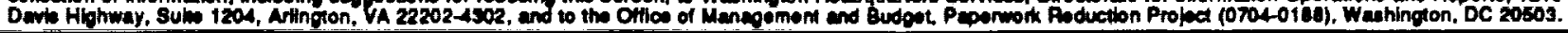
1. AOENCY USE ONLY (Lave blank)
2. AEPOAT DATE
3. REPORT TYPE AND DATES COVEAED
June 1992
Technical Memorandum

4. TITLE AND SUBTITLE

Vision-Based Range Estimation Using Helicopter Flight Data

6. AUTHOR(S)

$505-64-36$

Philip N. Smith, Banavar Sridhar, and Bassam Hussien

7. PEAFORIING OROANIZATION MAUE(8) AND ADDRESS(ES)

Ames Research Center

Moffett Field, CA $94035-1000$

- PERformino oroanization AEPOAT NUMBER

A-92085

0. SPONSOAINOMONITORING AGENCY MAME(S) AND ADDRESS(ES)

10. SPON8ORINGINONITORING AGENCY REPORT NUMBER

National Aeronautics and Space Administration

Washington, DC 20546-0001

NASA TM-103930

11. BUPPLEMENTAAY NOTES

Point of Contact: Philip N. Smith, Ames Research Center, MS 210-9, Moffett Field, CA 94035-1000

(415) $604-5469$ or FTS $464-5469$

12a. DISTRIBUTIOW/AVAILADILITY BTATEMENT

12b. DI8TAIBUTION CODE

Unclassified - Unlimited

Subject Category 04

13. ABsTRACT (Maximum 200 morde)

Pilot aiding during low-altitude flight depends on the ability to detect and locate obstacles near the helicopter's intended flightpath Computer-vision-based methods provide one general approach for obstacle detection and range estimation. Several algorithms have been developed for this purpose, but have not been tested with actual flight data. This paper presents results obtained using helicopter flight data with a feature-based range estimation algorithm. A method for recursively estimating range using a Kalman filter with a monocular sequence of images and knowledge of the camera's motion is described. The helicopter flight experiment and four resulting datasets are discussed. Finally the performance of the range estimation algorithm is explored in detail based on comparison of the range estimates with true range measurements collected during the flight experiment.

14. BUBJECT TERMS

16. NUMEER OF PAOES

Passive range estimation, Computer vision, Helicopter test flight

17. SECUAITY CLASSIFICATION OF REPOAT

Unclassified
18. SECURITY CLASSIFICATION OF THIS PAGE

Unclassified
19. SECURITY CLASSIFICATION OF ABSTRACT
20. LIMITATION OF ABSTRACT

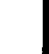

